ИЗВЕСТИЯ АКАДЕМИИ НАУК ЭСТОНСКОИ ССР. ТОМ 26 ФИЗИКА * МАТЕМАТИКА, 1977, № 2

Галина ПРИСТАВКО

\title{
ОПТИМАЛЬНАЯ ОСТАНОВКА СЛУЧАЙНЫХ ПРОЦЕССОВ
}

Galina PRISTAVKO. JUHUSLIKE PROTSESSIDE OPTIMAALNE PEATAMINE

Galina PRISTAVKO. ON OPTIMAL STOPPING FOR STOCHASTIC PROCESSES

Введение и постановка задачи. Рассмотрим на фиксированном вероятностном пространстве $(\Omega, F, P)$ непрерывный справа сепарабельный случайный процесс $X=\left(x_{t}, t \geqslant 0\right)$ со значениями в фазовом пространстве $(E, B)$, где $E-$ полукомпакт, $B$ - борелевская $\sigma$-алгебра, $F=$ $=\left\{F_{t}\right\}$ - неубывающее непрерывное справа семейство б-алгебр, такой, что $x_{t} F_{t}$-измеримы и $M x_{t}^{-}<\infty$, где $x^{-}=\max (0,-x)$.

Обозначим через $\bar{C}_{t}$ класс марковских моментов (м. м.) $\tau$ таких, что $\tau \geqslant t$ и $M x_{\tau}^{-}<\infty ; C_{t}-$ класс конечных м. м. из $\bar{C}_{t}$ (марковских моментов остановки (м. м.о.)); $L_{t}\left(\bar{L}_{t}\right)-$ класс м. м. $\tau$ из $C_{t}\left(\bar{C}_{t}\right)$, для которых $P\{\tau>t\}=1$.

Назовем «t-ценой» функцию $V_{t}=\sup _{\tau \in C_{t}} M x_{\tau}\left(W_{t}=\sup _{\tau \in L_{t}} M x_{\tau}\right)$. Известно $\left[{ }^{1}\right]$, что при сформулированных выше условиях существует обобщенный супермартингал $\left(c_{t}, F_{t}\right)\left(M c_{t}^{-}<\infty\right.$ и $M\left(c_{s} \mid F_{t}\right) \leqslant c_{t}$ при $s \leqslant t$ ) такой, что $V_{t}=M c_{t}$, где $\quad c_{t}=\operatorname{ess} \sup _{\tau \in c_{t}} M\left(x_{\tau} \mid F_{t}\right)$.

Обозначим $l_{t}=\underset{\tau \in L_{t}}{\operatorname{ess} \sup } M\left(x_{\tau} \mid F_{t}\right)$, тогда $W_{t}=M l_{t}$.

Будем говорить, что

1) момент $\tau_{0}(t)$ является $(0, t)$-оптимальным в классе $C_{t}\left(L_{t}\right)$, если $P$ - почти наверное (п. н.)

$$
M\left(x_{\tau_{0}(t)} \mid F_{t}\right)=c_{t} \quad\left(M\left(x_{\tau_{0}(t)} \mid F_{t}\right)=l_{t}\right) ;
$$

2) момент $\tau_{\varepsilon}(t)$ является $(\varepsilon, t)$-оптимальным в классе $C_{t}\left(L_{t}\right)$, если п. н.

$$
M\left(x_{\tau_{\varepsilon}(t)} \mid F_{t}\right) \geqslant c_{t}-\varepsilon \quad\left(M\left(x_{\tau_{\varepsilon}(t)} \mid F_{t}\right) \geqslant l_{t}-\varepsilon\right) ;
$$

3) момент $\sigma_{\varepsilon_{t}}(t)$ является $\left(\varepsilon_{t}, t\right)$-оптимальным в классе $C_{t}\left(L_{t}\right)$, где $\varepsilon_{t}$ некоторый случайный процесс, если п. н.

$$
M\left(x_{\sigma_{\varepsilon_{t}}}(t) \mid F_{t}\right) \geqslant c_{t}-\varepsilon_{t}\left(M\left(x_{\sigma_{\varepsilon_{t}}}(t) \mid F_{t}\right) \geqslant l_{t}-\varepsilon_{t}\right)
$$

(в наиболее интересных случаях $\varepsilon_{t} \equiv \varepsilon, \varepsilon_{t}=\varepsilon \cdot c_{t}\left(\varepsilon_{t}=\varepsilon \cdot l_{t}\right), \quad \varepsilon_{t}=$ $=\varepsilon \cdot\left[c_{t}-M\left(x_{\infty} \mid F_{t}\right)\right]\left(\varepsilon_{t}=\varepsilon \cdot\left[l_{t}-M\left(x_{\infty} \mid F_{t}\right)\right]\right)$, где $\left.x_{\infty}=\varlimsup_{t} x_{t}, \varepsilon>0\right)$.

В том случае, когда процесс $X=\left(x_{t}, t \geqslant 0\right)$ удовлетворяет условию $A^{-}: M\left(\sup _{t} x_{t}^{-}\right)<\infty$, процесс $c=\left(c_{t}, t \geqslant 0\right)$ является (см. [1]) наи- 
меньшим непрерывным справа $C_{t}$-регулярным супермартингалом (т. е. для любых $\sigma, \tau \in C_{t}$ с $P\{\sigma \leqslant \tau\}=1$ выполняется $\left.M c_{\tau} \leqslant M c_{\sigma}\right)$, мажорирующим $X=\left(x_{t}, t \geqslant 0\right)$, а при дополнительном условии $A^{+^{-}}: M\left(\sup _{t} x_{t}^{+}\right)<$ $<\infty$ моменты $\tau_{\varepsilon}(t)=\inf \left\{s \geqslant t: c_{s} \leqslant x_{s}+\varepsilon\right\}$ являются $(\varepsilon, t)$-оптимальными в классе $C_{t}$ и моменты $\tau_{0}(t)=\inf \left\{s \geqslant t: c_{s}=x_{s}\right\}$ являются $(0, t)$ оптимальными в классе $C_{t}$, если процесс $X=\left(x_{t}, t \geqslant 0\right)$ непрерывен с вероятностью 1.

В настоящей работе получен в определенном смысле окончательный результат, а именно дана (регулярная) характеризация цены в общем случае, а также показано существование $(\varepsilon, t)$-оптимальных (при условии $\left.A^{+*}\right)$ и $\left(\varepsilon_{t}, t\right)$-оптимальных моментов в общем случае для специальным образом подобранных процессов $\varepsilon t$.

В частном случае марковских процессов аналогичные вопросы рассматривались в $\left[{ }^{2}\right]$.

Случай $A^{+}$. Т еоре м а 1. Пусть случайный процесс $X=\left(x_{t}, t \geqslant 0\right)$ удовлетворяет условию $A^{+}$. Тогда

1) процесс $l=\left(l_{t}, t \geqslant 0\right)$ является наименьшим непрерывным справа $\bar{L}_{t}$-регулярным супермартингалом (т. е. для любых $\sigma, \tau \in \bar{L}_{t} \quad c$ $P\{\sigma \leqslant \tau\}=1$ выполняется $\left.M l_{\tau} \leqslant M l_{\sigma}\right)$, мажорирующим процесс $X=$ $=\left(x_{t}, t \geqslant 0\right)$;

2) $l_{t}=\bar{l}_{t}$, гдe $\bar{l}_{t}=$ ess sup $M\left(x_{\tau} \mid F_{t}\right)$.

$$
\tau \in \bar{L}_{t}
$$

Доказательство проводится с помощью «урезанных» снизу процессов $\quad l_{t}^{a^{7}}=\underset{\tau \in L_{t}}{\operatorname{ess} \sup } M\left(x_{\tau}^{a} \mid F_{t}\right), \quad$ где $\quad x_{t}^{a^{*}}=\max \left(a, x_{t}\right), \quad a \leqslant 0$, с последующим предельным переходом $a \rightarrow-\infty$. При этом устанавливается, что предельный процесс $l_{i}^{*}=\lim _{a \rightarrow-\infty} l_{t}^{a^{*}} \quad$ является непрерывным справа и совпалает п. н. с $l_{t}$.

Т еорем а 2. Пусть случайный процесс $X=\left(x_{t}, t \geqslant 0\right)$ удовлетвопяет условию $A^{+}$. Тогда

1) моменты $\tau_{\varepsilon}(t)=\inf \left\{s \geqslant t: t_{s} \leqslant x_{s}+\varepsilon\right\}$ являются при $\varepsilon>0(\varepsilon, t)$ оптимальными м. м. в классе $L_{+}$;

2) моменты $\tau_{0}(t)=\inf \left\{s \geqslant t: l=x_{2}\right\}$ являются $(0, t)$-оптимальными м. м. в классе $\bar{L}_{t}$, если проиесс $X=\left(x_{t}, t \geqslant 0\right)$ непрерывен с вероятностью 1:

3) если существует (0.t)-оптимальный м. м. $\tau^{*}(t) \in L_{t}\left(\bar{L}_{t}\right)$. то момент $\tau_{0}(t)$ является также $(0, t)$-оптимальным в классе $L_{t}\left(\bar{L}_{t}\right)$ u $P\left\{\tau_{0}(t) \leqslant\right.$ $\left.\leqslant \tau^{*}(t)\right\}=1$.

Общий случай. Т еорем а 3 . Пусть $X=\left(x_{t}, t \geqslant 0\right)-$ случайный процесс. Тогда

1) процесс $l=\left(l_{t}, t \geqslant 0\right)$ является наименьшим непрерывным справа $L_{+}$-регчлярным супермартингалом (т. е. для любых $\sigma, \tau \in L_{+} \quad c$ $P\{\sigma \leqslant \tau\}=1$ выполняется $\left.M l_{\tau} \leqslant M l_{\sigma}\right)$, мажорирующим процесс $X=$ $=\left(x_{t}, t \geqslant 0\right)$.

2) $l_{t}=\bar{l}_{t}$.

Доказательст во проводится с помощью «урезанных» сверху процессов $\quad l_{t}^{b^{a}}=\underset{\tau \in L_{t}}{\operatorname{ess} \sup } M\left(x_{\tau}^{b} \mid F_{t}\right)$, где $x_{t}^{b^{-}}=\min \left(b, x_{t}\right), b \geqslant 0, \quad$ с последуюшим предельным переходом $b \rightarrow \infty$.

Обобщением теппемы 3 является следующий результат.

Т еорема 4. Пусть $X=\left(x_{t}, t \geqslant 0\right)$ - cлучайный процеес. Обозначим 


$$
\hat{l}_{\tau}= \begin{cases}\frac{l_{t}(\omega)}{\lim _{t} x_{t},}, \omega \in\{\omega: \tau=t\}\end{cases}
$$

Тогда для любых м. м. $\tau, \sigma \in \bar{L}_{t}$ с $P\{\sigma \leqslant \tau\}=1$ выполняется $M \hat{l}_{\tau} \leqslant$ м $\hat{l}_{\sigma}$ (т. е. $\hat{l}_{t}$ является $\bar{L}_{t}$-регулярным).

Т еорема 5. Пусть $X=\left(x_{t}, t \geqslant 0\right)$ - случайный процесс $u l_{t}=$ $=\underset{\tau \in L_{t}}{\operatorname{ess} \sup } M\left(x_{\tau} \mid F_{t}\right)$ конечно. Тогда

$$
\tau \in L_{t}
$$

если существует $(0, t)$-оптимальный $м . \quad$ м. $\tau^{*}(t) \in L_{t}\left(\bar{L}_{t}\right)$, то момент $\tau_{0}(t)$ является также $(0, t)$-оптимальным в классе $L_{t}\left(\bar{L}_{t}\right)$ и $P\left\{\tau_{0}(t) \leqslant\right.$ $\left.\leqslant \tau^{*}(t)\right\}=1$.

Т ео рем а 6 . Пусть случайный процесс $X=\left(x_{t}, t \geqslant 0\right)$ удовлетворяет условию $a^{-}: M\left(\overline{\lim x_{t}}\right)<\infty, \tilde{\varepsilon}_{t}=\varepsilon \cdot \tau_{t}$, где $\tau_{t}=l_{t}-M\left(\underset{\lim x_{s}}{ } \mid F_{t}\right)$, $\varepsilon>0, t \in T_{\tilde{\alpha}}=\left\{t: l_{t}<\infty\right\}$. Тогда моменты $\sigma_{\tilde{\varepsilon}_{t}}(t)=\inf \left\{s \geqslant t: l_{s} \leqslant x_{s}+\tilde{\varepsilon_{c}}\right\}$ являются $\left(\tilde{\varepsilon}_{t}, t\right)$-оптимальными м. м. в классе $\bar{L}_{t}$ для $t \in T_{0}$.

Д ок а з те льст в о основывается на следующей теореме.

Теорема 7. Пусть $X=\left(x_{t}, t \geqslant 0\right)$ - случайный процесс, $\varepsilon_{t}=$ $=\varepsilon \cdot l_{t}, \quad \varepsilon>0, \quad t \in T_{0}, \quad P\left\{\overline{\lim }_{t} x_{t} \geqslant 0\right\}=1$. Тогда моменты $\sigma_{\varepsilon_{t}}(t)=$ $=\inf \left\{s \geqslant t: l_{s} \leqslant x_{s}+\varepsilon_{s}\right\}$ являются $\left(\varepsilon_{t}, t\right)$-оптимальными в классе $\bar{L}_{t}$. Доказ ательст во этой теоремы опирается на следующую лемму, представляющую и самостоятельный интерес.

Л ем м а. Пусть $X=\left(x_{t}, t \geqslant 0\right)$ - случайный процесс $u$ $P\left\{\overline{\lim }_{t} x_{t} \geqslant 0\right\}=1$. Tогда $l_{t}=M\left(I_{\left\{\sigma_{\varepsilon_{t}}(t)<\infty\right\}} l_{\sigma_{\varepsilon_{t}}(t)}\left|F_{-}\right|+M\left(I_{\left\{\sigma_{\varepsilon_{t}}(t)=\infty\right\}} \overline{\left.\lim _{s} x_{s} \mid F_{t}\right)}\right.\right.$, где $I_{A}(x)=\left\{\begin{array}{l}1, x \in A \\ 0, x \notin A .\end{array}\right.$

\section{Л И ТЕРАТ У РА}

1. Ф.окее в А. Г., Теория вероятности и ее применения, XV, № 2 (1970).

2. Шир я в А. Н., Статистический последовательный анализ, М., 1976.

Ннститут кибернетики Академии наук Эстонской ССР
Поступила в редакцию 15/VII 1976 